\title{
Predicting resistance-AR-V7 is a potential biomarker
}

The detection of androgen-receptor splice variant 7 (AR-V7) in circulating tumour cells from men with metastatic castrationresistant prostate cancer (mCRPC) could predict patient response to treatment with either enzalutamide or abiraterone, according to a new report in The New England Journal of Medicine.

\section{No patient who tested positive for AR-V7 at baseline [benefited]... 77}

This study prospectively enrolled 62 patients who were beginning treatment for mCRPC; 31 men were assigned to receive $160 \mathrm{mg}$ enzalutamide daily and another 31 patients were given daily doses of 1,000 mg abiraterone. Participants were assessed for baseline levels of both full-length androgen receptor and AR-V7 mRNA in circulating tumour cells collected from a peripheral blood sample.

The primary end point of this study was the proportion of patients who exhibited a PSA response, defined as a $\geq 50 \%$ decline in serum PSA level at baseline, maintained for 4 weeks. Secondary end points were progression-free survival (PFS) with regards to serum PSA, clinical or radiographic progression and overall survival.

In the enzalutamide treatment arm, 39\% of patients tested positive for AR-V7, and AR-V7 was detected in $19 \%$ of participants in the abiraterone treatment group before they commenced treatment.

Lower serum PSA response rates were observed in patients positive for AR-V7 in both treatment groups compared with AR-V7-negative participants $(0 \%$ versus $53 \%, P=0.004$ for enzalutamide-treated men, and $0 \%$ versus $68 \%, P=0.004$ for abiraterone-treated men). The authors report that higher levels of full-length androgen receptor mRNA were associated with positive AR-V7 status, but the presence of AR-V7 remained predictive of serum PSA response to treatment after adjustment for full-length androgen receptor expression $(P=0.02)$.
Patients who were positive for AR-V7 also had shorter serum PSA PFS, clinical and radiographic PFS and overall survival. The effect of positive AR-V7 status was maintained in a combined analysis of the secondary end points across both treatment groups in all 62 participants $(P<0.001)$.

No patient who tested positive for AR-V7 at baseline demonstrated a clinical benefit from treatment with enzalutamide or abiraterone in this study. Large-scale prospective studies are required to validate the findings of this small study, but if these results are confirmed, AR-V7 could be used as a biomarker for resistance to enzalutamide and abiraterone therapy. This information might help inform clinicians about the best course of treatment for patients with $\mathrm{mCRPC}$.

Louise Stone

Original article Antonarakis, E. et al. AR-V7 and resistance to enzalutamide and abiraterone in prostate cancer. N. Engl. J. Med. doi:10.1056/NEJMoa1315815 